Ferrata Storti Foundation

\title{
Genomic landscape of pediatric B-other acute lymphoblastic leukemia in a consecutive European cohort
}

Haematologica 2019

Volume 104(7):1396-1406

\section{Correspondence:}

JAN TRKA/MARKETA ZALIOVA jan.trka@lfmotol.cuni.cz/ marketa.zaliova@|fmotol.cuni.cz

Received: August 21, 2018.

Accepted: December 20, 2018.

Pre-published: January 10, 2019.

doi:10.3324/haematol.2018.204974

Check the online version for the most updated information on this article, online supplements, and information on authorship \& disclosures: www.haematologica.org/content/104/7/1396

\section{(C)2019 Ferrata Storti Foundation}

Material published in Haematologica is covered by copyright. All rights are reserved to the Ferrata Storti Foundation. Use of published material is allowed under the following terms and conditions:

https://creativecommons.org/licenses/by-nc/4.0/legalcode. Copies of published material are allowed for personal or internal use. Sharing published material for non-commercial purposes is subject to the following conditions:

https://creativecommons.org/licenses/by-nc/4.0/legalcode, sect. 3. Reproducing and sharing published material for commercial purposes is not allowed without permission in writing from the publisher.

\author{
Marketa Zaliova, ${ }^{1,2,3}$ Jan Stuchly, ${ }^{1,2}$ Lucie Winkowska, ${ }^{1,2}$ Alena Musilova, ${ }^{1,2}$ \\ Karel Fiser, ${ }^{1,2}$ Martina Slamova, ${ }^{1,2}$ Julia Starkova, ${ }^{1,2}$ Martina Vaskova,,$^{1,2}$ \\ Ondrej Hrusak, ${ }^{1,2,3}$ Lucie Sramkova, ${ }^{1,2,3}$ Jan Stary, ${ }^{2,3}$ Jan Zuna ${ }^{1,2,3}$ and Jan Trka ${ }^{1,2,3}$ \\ ${ }^{1}$ CLIP - Childhood Leukaemia Investigation Prague; ${ }^{2}$ Department of Paediatric \\ Haematology and Oncology, Second Faculty of Medicine, Charles University and \\ ${ }^{3}$ University Hospital Motol, Prague, Czech Republic
}

\section{ABSTRACT}

$\mathrm{N}$ ovel biological subtypes and clinically important genetic aberrations (druggable lesions, prognostic factors) have been described in Bother acute lymphoblastic leukemia (ALL) during the last decade; however, due to a lack of studies on unselected cohorts, their population frequency and mutual associations still have to be established. We studied 110 consecutively diagnosed and uniformly treated childhood B-other patients using single nucleotide polymorphism arrays and whole exome/transcriptome sequencing. The frequency of DUX4-rearranged, $B C R$-ABL1-like, ZNF384-rearranged, ETV6-RUNX1-like, iAMP21 and MEF2D-rearranged subtypes was $27 \%, 15 \%, 5 \%, 5 \%, 4 \%$, and $2 \%$, respectively; $43 \%$ of cases were not classified into any of these subtypes (B-rest). We found worse early response to treatment in DUX4-rearranged leukemia and a strong association of ZNF384-rearranged leukemia with B-myeloid immunophenotype. Of the druggable lesions, JAK/STAT-class and RAS/RAF/MAPK-class aberrations were found in $21 \%$ and $43 \%$ of patients, respectively; an ABL-class aberration was found in one patient. A recently described negative prognostic factor, $I K Z F 1^{\text {plus }}$, was found in $14 \%$ of patients and was enriched in (but not exclusive for) $B C R$ - $A B L 1$-like subtype. PAX5 fusions (including 4 novel), intragenic amplifications and P80R mutations were mutually exclusive and only occurred in the B-rest subset, altogether accounting for $20 \%$ of the B-other group. PAX5 P80R was associated with a specific gene expression signature, potentially defining a novel leukemia subtype. Our study shows unbiased European population-based frequencies of novel ALL subtypes, recurrent (cyto)genetic aberrations and their mutual associations. This study also strengthens and widens the current knowledge of B-other ALL and provides an objective basis for optimization of current genetic diagnostics.

\section{Introduction}

Knowledge about the genetics of pediatric B-cell precursor (BCP) acute lymphoblastic leukemia (ALL) has grown substantially in the past decade due to the boom in genome-wide technologies. Some of the most important novel findings concern B-other ALL, a heterogeneous group of BCP-ALL with previously unknown genetic backgrounds, usually defined by the absence of all routinely assessed classifying aberrations. Several subtypes have been described within Bother ALL based on the presence of unique (presumably primary) genetic aberrations [iAMP21 ALL, ${ }^{1}$ DUX4 rearranged (r) ALL, ${ }^{2}$ ZNF384r ALL, ${ }^{3,5,6}$ MEF2Dr ALL $\left.{ }^{3,6,7}\right]$ or gene expression signatures (BCR-ABL1-like ALL-10 and ETV6-RUNX1-like $\left.\mathrm{ALL}^{2,11}\right)$. Although the classification of BCP-ALL has been substantially refined, it is complicated by a partial overlap of some of these subtypes and/or their imperfect definitions. It has been shown that a significant proportion of AAMP21 ALL have 
Table 1. Early response to treatment of B-other acute lymphoblastic leukemia patients stratified by DUX4r.

$\begin{array}{cccc}\text { Total B-other } & \text { DUX4r ALL } & \text { non-DUX4r ALL } & P \\ (n=1,0) & (n=30) & (n=80) & \text { DUX4r vs. non-DUX4r } \\ n(\%) & n(\%) & n\end{array}$

Prednisone response ${ }^{1}$

\begin{tabular}{lcccc} 
Good & $102(93 \%)$ & $24(80 \%)$ & $78(98 \%)$ & \\
Poor & $8(7 \%)$ & $6(20 \%)$ & $2(2 \%)$ & 0.005 \\
FC MRD d15 & & & & \\
$<10 \%$ & $86(82 \%)$ & $19(68 \%)$ & $67(87 \%)$ & 0.04 \\
$\geq 10 \%$ & $19(18 \%)$ & $9(32 \%)$ & $10(13 \%)$ & 3 \\
NA & 5 & 2 & & \\
\hline
\end{tabular}

\section{PCR MRD ${ }^{3}$}

\begin{tabular}{|c|c|c|c|c|}
\hline TP1 + TP2 neg. & $28(26 \%)$ & $3(10 \%)$ & $25(32 \%)$ & \multirow{4}{*}{0.04} \\
\hline Other & $56(53 \%)$ & $17(59 \%)$ & $39(51 \%)$ & \\
\hline TP1 $\geq 10-3+$ TP2 pos. or TP2 $\geq 10-3$ & $22(21 \%)$ & $9(31 \%)$ & $13(17 \%)$ & \\
\hline $\mathrm{NA}$ & $4^{5}$ & 1 & 3 & \\
\hline \multicolumn{5}{|l|}{ Final risk group ${ }^{4}$} \\
\hline SR & $25(23 \%)$ & $3(10 \%)$ & $22(28 \%)$ & \multirow{4}{*}{0.03} \\
\hline MR & $52(49 \%)$ & $13(45 \%)$ & $39(50 \%)$ & \\
\hline HR & $30(28 \%)$ & $13(45 \%)$ & $17(22 \%)$ & \\
\hline NA & $3^{5}$ & 1 & 2 & \\
\hline
\end{tabular}

${ }^{1}$ Good: $<1000$ leukemic blood blasts/ $\mu \mathrm{L}$ on treatment day (d) 8; poor: $\geq 1000 / \mu \mathrm{L} .{ }^{2}$ Minimal residual disease (MRD) at d15 of treatment measured by flow cytometry (FC). ${ }^{3} \mathrm{MRD}$ measured by polymerase chain reaction (PCR). n: number; TP: time point; TP1: treatment day 33;TP2: treatment day 78. ${ }^{4}$ Standard risk (SR), medium risk (MR), high risk (HR); for definitions see Online Supplementary Methods. P-value by Fisher exact test. ${ }^{5}$ Patients died before TP2 or no MRD target; neg.: negative; pos.: positive (any positive value); NA: not applicable/not available. Unknown values are not considered for percentage and statistics.

the $B C R-A B L 1$-like phenotype ${ }^{12}$ or may coincide with the ETV6-RUNX1-like phenotype. ${ }^{11}$ Moreover, it has been demonstrated that the definition of BCR-ABL1-like ALL varies significantly among studies; ${ }^{12}$ this likely results from the limited specificity of $B C R-A B L 1$-associated gene expression signature. Thus, not only a complex genetic and gene expression characterization but also a hierarchical approach is needed in order to classify BCP-ALL into non-overlapping subsets.

In parallel to aberrations that define subtypes, a wide range of additional genetic aberrations has been identified. Among them, two groups of aberrations have already become therapeutically relevant either as novel prognostic factors or as therapy targets. The first group is represented by IKZF1 gene deletion (IKZF1del) and IKZF1 plus deletion pattern. IKZF1 del was repeatedly shown to confer a higher risk of relapse $e^{9,13-15}$ and is used in therapy adjustment algorithms in some contemporary treatment protocols (clinicaltrials.gov identifier: 03020030; DCOG ALL11 ${ }^{16}$ ). However, the Italian Association of Pediatric Hematology and Oncology-Berlin-Frankfurt-Muenster (AIEOP-BFM) study group has recently shown that $I K Z F$ plus $^{\text {plu }}$, defined by a combination of $I K Z F 1$ del with other aberrations (deletions of CDKN2A,CDKN2B, PAX5, or PAR1 in the absence of $E R G$ deletion), confers a significantly higher risk of relapse compared to IKZF1del alone, and the newly defined $I K Z F 1^{\text {plus }}$ pattern will be used to improve risk prediction accuracy in the upcoming BFM ALL protocol. ${ }^{17}$ The second group of therapeutically relevant aberrations largely comprises aberrations affecting genes encoding kinases, cytokine receptors and signaling regulators, resulting in activation of kinase signaling ${ }^{10,18-23}$ ("kinase aberrations"). These aberrations occur especially, but not exclusively, in $B C R$ - $A B L 1$-like ALL. A wide range of kinase aberrations has already been described, and novel ones still continue to be reported. These aberrations vary by type (gene fusions/juxtapositions, copy number changes, mutations) and frequency; CRLF2, NRAS or KRAS lesions are relatively common and occur in various ALL subtypes, ${ }^{2,6,24-27}$ while, for example, fusions involving $A B L 1$, $A B L 2, E P O R, P D G F R B$ or $J A K 2$ are less frequent and occur predominantly in $B C R-A B L 1$-like ALL. ${ }^{28-30}$ Two functional classes of aberrations, ABL-class and JAK/STAT-class, are currently used for targeted ALL therapy in some ongoing clinical trials (clinicaltrials.gov identifiers: 03117751, 02883049, 03020030).

The ongoing translation of these genetic findings into treatment is bringing new diagnostic demands and a dilemma as to which genetic methods should be incorporated into diagnostic algorithms and how this should be achieved. In order to explore this, well-defined consecutive cohorts need to be analyzed to assess the real incidence of various ALL subtypes and genetic aberrations. Unfortunately, so far, such data have been very scarce, and virtually all the published cohorts were in some way selected, enriched, biased, or not analyzed completely.

Here, we present a genetic study of all pediatric B-other ALL cases consecutively diagnosed and uniformly treated in the Czech Republic over the last seven years. We performed this study to facilitate the use of optimal treatment strategies by extending and refining diagnostic algorithms, and to assess the feasibility and the benefits of introducing genome-wide technologies into routine diagnostics. A further study aim was to determine unbiased population frequencies of known ALL subtypes derived from B-other 
A A HCA with DUX4r ALL specific gene set

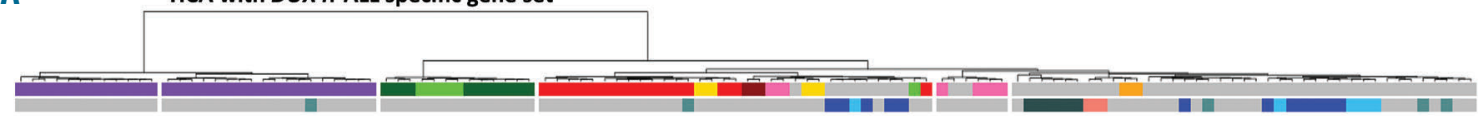

B

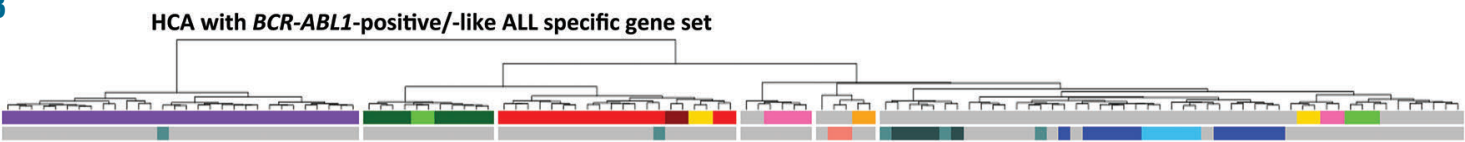

C HCA with ETV6-RUNX1-positive/-like ALL specific gene set

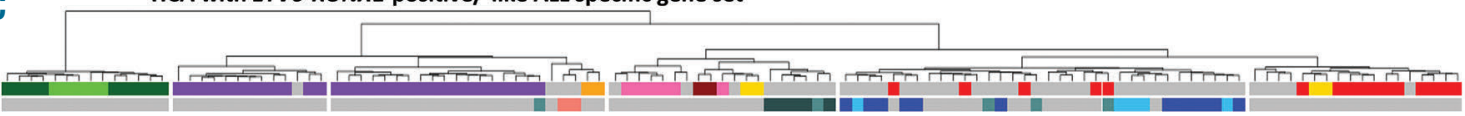

D Unsupervised HCA

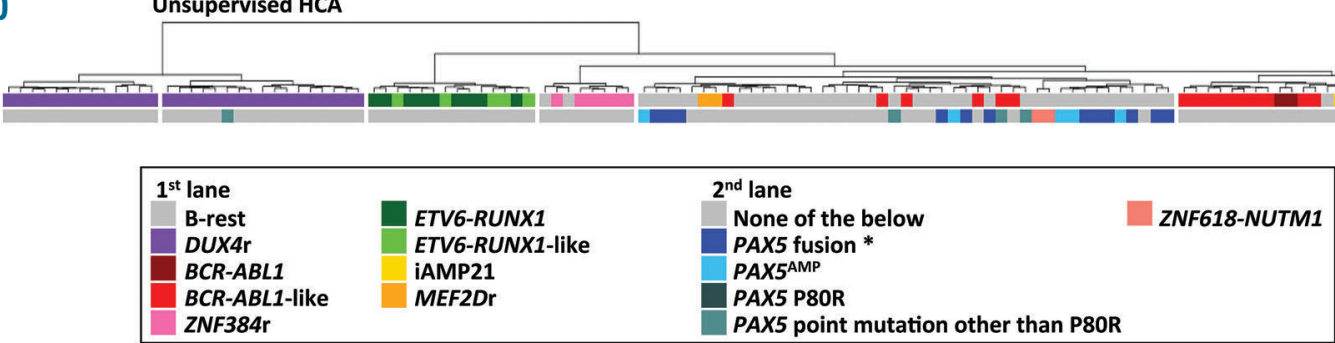

Figure 1. Results of hierarchical clustering analyses (HCA). A total of 110 B-other acute lymphoblastic leukemia (ALL) were clustered hierarchically based on the expression of genes belonging to defined gene sets (A-C) or based on the expression of the most variably expressed transcripts (409 transcripts with standard deviations $\geq 35 \%$ of the maximal standard deviation) (D) and resulting dendrograms are shown. Gene sets are described in Online Supplementary Tables S4-S5. Genetic annotation is split in two lanes: $1^{\text {st }}$ lane shows classification into already established subtypes, $2^{\text {nd }}$ lane shows selected recurrent genetic aberrations (mutually exclusive with each other and with the exception of PAX5 mutations other than P8OR also with established subtypes). *Does not include ZCCHC7-PAX5 fusion.

ALL and genetic aberrations, their mutual associations, and associations with demographic and clinical parameters.

\section{Methods}

\section{Patients}

The present study included 110 children (aged 1-18 years) diagnosed with B-other ALL [BCP-ALL negative for ETV6-RUNX1, $B C R-A B L 1, T C F 3-P B X 1, K M T 2 A$-rearrangements, hyperdiploidy ( $>50$ chromosomes), and hypodiploidy ( $<44$ chromosomes)] between December 2010 and December 2017 treated according to the AIEOP-BFM ALL 2009 trial (clinicaltrials.gov identifier: 01117441). In total, 410 BCP-ALL and/or BCP-myeloid mixed phenotype acute leukemia (MPAL) cases were diagnosed and treated according to this protocol within the period of study (Online Supplementary Table S1). The study was approved by the local Institutional Review Board. Informed consent was obtained in accordance with the Declaration of Helsinki.

\section{Analysis of ERG gene deletion and P2RY8-CRLF2 by polymerase chain reaction}

Presence of the ERG gene deletion (ERGdel) was analyzed by DNA-based multiplex polymerase chain reaction (PCR). ${ }^{31}$ Two primers corresponding to additional centromeric breakpoint sites ${ }^{32}$ were added: 5'-GCGGCTACTTGTTGGTCCAAGAA-3' and 5'CTATCCTGAACATTGCTGCCAG-3'.

Presence of the P2RY8-CRLF2 transcript was analyzed by real time (RT)-PCR and quantified by quantitative real time (qRT)-PCR as described previously ${ }^{18}$ with replacement of the reverse primer for qRT-PCR (5'-AGCCTCCCAGCAGAAAGAC-3').

\section{Single-nucleotide polymorphism array}

Single-nucleotide polymorphism array ( $\mathrm{SNPa}$ ) was performed in 103 out of 110 B-other patients. Copy number aberrations (CNA) and regions of uniparental disomy (UPD) were identified using HumanOmni Express BeadChip (Illumina, USA) or CytoScan HD arrays (Affymetrix, USA).

\section{Whole-transcriptome and whole-exome sequencing}

Whole-transcriptome (RNA-sequencing) and whole-exome (WES) sequencing were performed using diagnostic samples in 109 out of 110 and 69 out of 110 B-other patients, respectively, as described previously. ${ }^{11}$ In one patient without material from initial diagnosis, the relapse sample was used for subtype classification. Sequencing libraries were prepared from DNA and total RNA using Agilent SureSelectXT HumanAllExon V4, V5 or V6 and Agilent SureSelect mRNA Strand Specific kits, respectively, according to the manufacturer's instructions (Agilent Technologies Inc., Santa Clara, CA, USA). High-throughput sequencing was performed on HiSeq2500 (1x50, 2x50 or $2 \times 100$ bp) or NextSeq500 (2x75 bp) using TruSeq Rapid SBS and PE Cluster kits and High Output Kit (Illumina).

Selected data metrics are shown in Online Supplementary Tables $S 2$ and $S 3$.

\section{Analysis of fusion transcripts and IGH, DUX4 and CRLF2 rearrangements}

RNA-sequencing data were analyzed using $\mathrm{TopHat}^{33}$ and deFuse. ${ }^{34}$ In-frame fusion transcripts and those out-of-frame fusion transcripts that disrupted known ALL-associated genes and/or correlated with boundaries of CNAs found by SNPa were selected for further verification. Fusions verified by RT-PCR as leukemia-spe- 


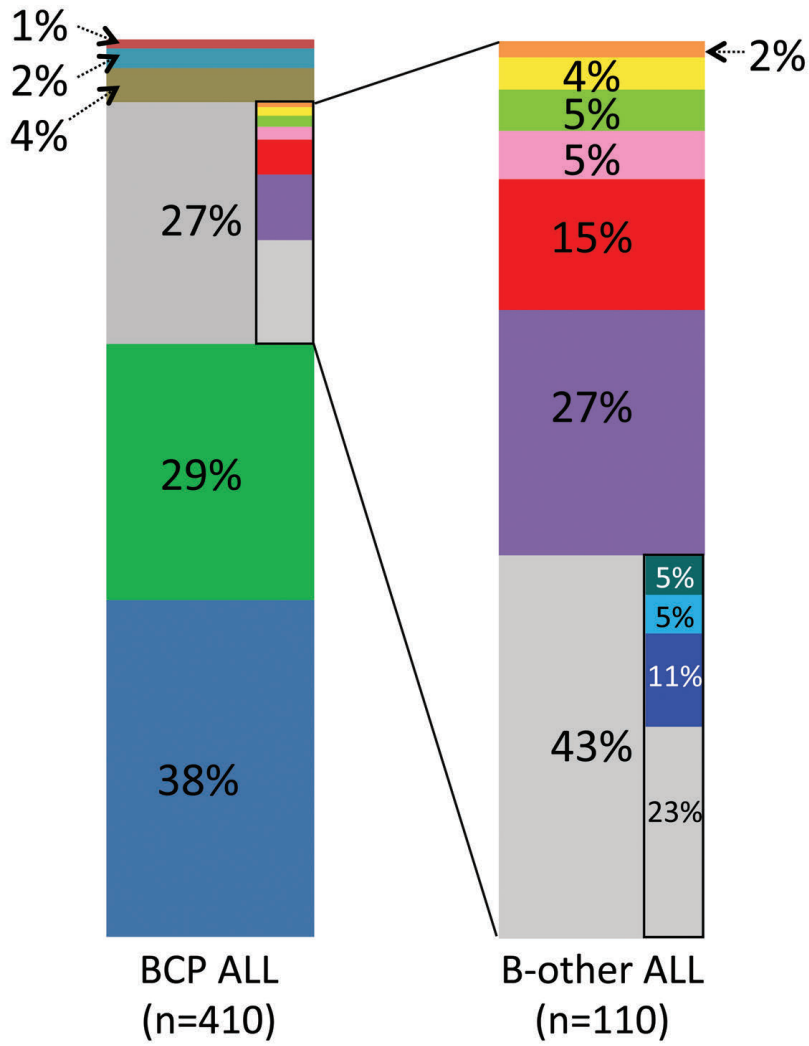

cific (absent in healthy mononuclear blood cells) are reported.

To analyze the presence of IGH-DUX4 and IGH-CRLF2 fusion transcripts that may not be revealed by TopHat/deFuse, reads mapped to fusion partner genes were analyzed again manually.

\section{Analysis of genomic variants}

Read pairs were aligned to hg19 using BWA $^{35}$ (WES) and STAR ${ }^{36}$ (RNA-sequencing) and further processed by Picard tools (http://broadinstitute.github.io/picard/). Variant calling was performed using VarScan ${ }^{37}$ and Samtools (http://samtools.sourceforge.net). To distinguish somatic and germline variants, WES results from ALL diagnosis were compared to the remission sample.

\section{Gene expression profiling, hierarchical clustering analysis, DUX4 gene expression analysis}

Genome-wide gene expression analysis was performed using RNA-sequencing data of the $110 \mathrm{~B}$-other study patients plus two $B C R-A B L 1$-positive and nine ETV6-RUNX1-positive patients. Alignment and counting were performed as described previously. ${ }^{38}$ Data normalization and hierarchical clustering analysis (HCA) based on the expression of the most variably expressed genes or of genes belonging to particular gene sets were performed using $\mathrm{R}$ package Deseq2 $2^{39}$ (vst normalization, ward.D method and Euclidean distance linkage for HCA). The list of gene sets and included genes used for HCA are shown in Online Supplementary Tables $S 4$ and $S 5$.

To assess DUX4 gene expression, reads mapped to the DUX4 reference were counted as described previously. ${ }^{3}$ To correct for uneven sequencing depth, counts were normalized using size factors computed by Deseq2.

\section{Flow cytometry}

Routine flow cytometry was used for the diagnosis of BCP-ALL and mixed-phenotype acute leukemia (MPAL) using criteria of the
Left graph

- Hypodiploidy

KMT2Ar

TCF3-PBX1

B-other

- ETV6-RUNX1

- Hyperdiploidy (>50 chr)

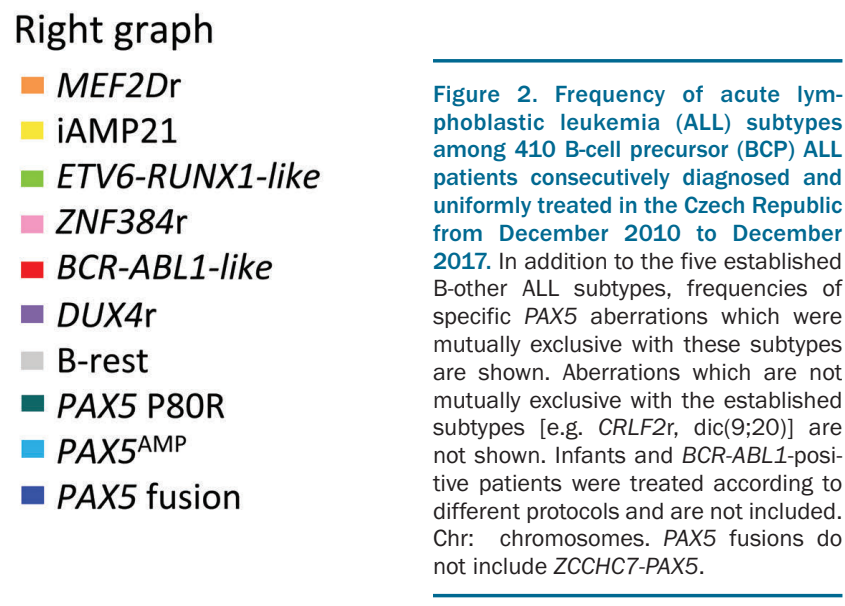

World Health Organization and/or of the European Group for Immunophenotyping of Leukemia. CRLF2 expression was assessed using PE anti-human TSLPR Antibody (clone 1D3; BioLegend, USA). In general, a $50 \%(10 \%)$ cut-off was used to assign strong (weak) positivity. A small CRLF2-positive subclone $(<10 \%)$, distinct from the major negative population and clearly distinguishable from the technical background, was reported in one patient.

For more details of the methods used see the Online Supplementary Methods.

\section{Results}

Frequency of the B-other-derived acute lymphoblastic leukemia subtypes and associations with clinical parameters

To classify B-other patients into iAMP21, ZNF384r, MEF2Dr, DUX4r, ETV6-RUNX1-like and BCR-ABL1-like subtypes, we used data from SNPa and RNA-sequencing. Our cohort included six cases with ZNF384 fusions (EP300-ZNF384, $\mathrm{n}=4$; TCF3-ZNF384, $\mathrm{n}=1$; TAF15ZNF384, n=1), four cases with iAMP21, and two cases with MEF2Dr (MEF2D-BCL9, $\mathrm{n}=2$ ). The BCR-ABL1-like, ETV6-RUNX1-like and DUX4r ALLs were classified using $\mathrm{HCA}$ based on the expression of gene sets representative of subtype-specific gene expression signatures (subtypespecific gene sets) from published studies ${ }^{4,10,11}$ (Figure 1AC). Using the DUX4r gene set, 30 cases were classified as DUX4r ALL. In accordance with published studies, DUX4r patients had significantly higher DUX4 expression compared to remaining ALLs (Online Supplementary Figure S1) and fusion transcripts supporting the presence of DUX4r were found in 29 out of 30 patients. To verify co-clustering 
Table 2. Frequency of selected genetic lesions in total cohort and across acute lymphoblastic leukemia subsets.

\begin{tabular}{|c|c|c|c|c|c|c|c|c|}
\hline & & Percenta & $\begin{array}{r}\text { tage of (number o } \\
\text { total B-0 }\end{array}$ & $\begin{array}{l}\text { f total analyzed) } \\
\text { other cohort / inc }\end{array}$ & $\begin{array}{l}\text { cases positiv } \\
\text { dividual ALL s }\end{array}$ & $\begin{array}{l}\text { for genetic } \\
\text { bsets }\end{array}$ & in & \\
\hline & $\begin{array}{c}\text { Total } \\
(n=103-110 t)\end{array}$ & $\begin{array}{l}\text { B-rest } \\
(n=47)\end{array}$ & $\begin{array}{l}\text { DUX4r } \\
(n=30)\end{array}$ & $\begin{array}{c}\text { BCR-ABL1-Hke } \\
(n=16)\end{array}$ & $\begin{array}{c}\text { ZNF384r } \\
\quad(n=6)\end{array}$ & $\begin{array}{c}\text { ETV6-RUNX1-Hke } \\
\qquad(n=5)\end{array}$ & $\begin{array}{l}\text { e IAMP21 } \\
\qquad(n=4)\end{array}$ & $\begin{array}{c}\text { MEF2Dr } \\
(n=2)\end{array}$ \\
\hline PAX5 any aberration & $53 \%(55 / 104)$ & $82 \%(36 / 44)^{* * * *}$ & * $\quad 21 \%(6 / 28)$ & $50 \%(8 / 16)$ & $17 \%(1 / 6)$ & $40 \%(2 / 5)$ & $33 \%(1 / 3)$ & $50 \%(1 / 2)$ \\
\hline PAX5 fusion ${ }^{1}$ & $11 \%(12 / 109)$ & $26 \%(12 / 47)^{* * * *}$ & $0 \%(0 / 30)$ & $0 \%(0 / 16)$ & $0 \%(0 / 6)$ & $0 \%(0 / 5)$ & $0 \%(0 / 3)$ & $0 \%(0 / 2)$ \\
\hline PAX5 AMP & $5 \%(5 / 109)$ & $11 \%(5 / 47)^{*}$ & $0 \%(0 / 30)$ & $0 \%(0 / 16)$ & $0 \%(0 / 6)$ & $0 \%(0 / 5)$ & $0 \%(0 / 3)$ & $0 \%(0 / 2)$ \\
\hline PAX5 P80R & $5 \%(5 / 109)$ & $11 \%(5 / 47)^{*}$ & $0 \%(0 / 30)$ & $0 \%(0 / 16)$ & $0 \%(0 / 6)$ & $0 \%(0 / 5)$ & $0 \%(0 / 3)$ & $0 \%(0 / 2)$ \\
\hline$C D K N 2 A B$ deletion & $51 \%(53 / 103)$ & $72 \%(31 / 43)^{* * *}$ & $32 \%(9 / 28)$ & $50 \%(8 / 16)$ & $33 \%(2 / 6)$ & $20 \%(1 / 5)$ & $33 \%(1 / 3)$ & $50 \%(1 / 2)$ \\
\hline $\operatorname{dic}(9 ; 20)$ & $9 \%(9 / 103)$ & $19 \%(8 / 43)^{* *}$ & $0 \%(0 / 28)$ & $6 \%(1 / 16)$ & $0 \%(0 / 6)$ & $0 \%(0 / 5)$ & $0 \%(0 / 3)$ & $0 \%(0 / 2)$ \\
\hline TOX deletion & $8 \%(8 / 103)$ & $19 \%(8 / 43)^{* * *}$ & $0 \%(0 / 28)$ & $0 \%(0 / 16)$ & $0 \%(0 / 6)$ & $0 \%(0 / 5)$ & $0 \%(0 / 3)$ & $0 \%(0 / 2)$ \\
\hline trisomy 5 & $4 \%(4 / 103)$ & $9 \%(4 / 43)^{*}$ & $0 \%(0 / 28)$ & $0 \%(0 / 16)$ & $0 \%(0 / 6)$ & $0 \%(0 / 5)$ & $0 \%(0 / 3)$ & $0 \%(0 / 2)$ \\
\hline$E R G$ deletion ${ }^{2}$ & $18 \%(19 / 108)$ & $0 \%(0 / 46)$ & $63 \%(19 / 30)^{* * * *}$ & $0 \%(0 / 16)$ & $0 \%(0 / 6)$ & $0 \%(0 / 5)$ & $0 \%(0 / 3)$ & $0 \%(0 / 2)$ \\
\hline TBLIXRI any aberration & $12 \%(12 / 103)$ & $5 \%(2 / 43)$ & $24 \%(7 / 29)^{*}$ & $6 \%(1 / 16)$ & $0 \%(0 / 6)$ & $20 \%(1 / 5)$ & $0 \%(0 / 3)$ & $50 \%(1 / 2)$ \\
\hline$D M D$ deletion & $6 \%(6 / 103)$ & $2 \%(1 / 43)$ & $18 \%(5 / 28)^{* *}$ & $0 \%(0 / 16)$ & $0 \%(0 / 6)$ & $0 \%(0 / 5)$ & $0 \%(0 / 3)$ & $0 \%(0 / 2)$ \\
\hline CRLF2 fusion $^{3}$ & $24 \%(26 / 109)$ & $28 \%(13 / 47)$ & $0 \%(0 / 30)$ & $69 \%(11 / 16)^{* * * *}$ & $0 \%(0 / 6)$ & $20 \%(1 / 5)$ & $33 \%(1 / 3)$ & $0 \%(0 / 2)$ \\
\hline CRLF2r & $15 \%(16 / 109)$ & $6 \%(3 / 47)$ & $0 \%(0 / 30)$ & $69 \%(11 / 16)^{* * * *}$ & $0 \%(0 / 6)$ & $20 \%(1 / 5)$ & $33 \%(1 / 3)$ & $0 \%(0 / 2)$ \\
\hline P2RY8-CRLF2-low & $9 \%(10 / 108)$ & $21 \%(10 / 47)^{* * *}$ & $0 \%(0 / 29)$ & $0 \%(0 / 15)$ & $0 \%(0 / 6)$ & $0 \%(0 / 5)$ & $0 \%(0 / 4)$ & $0 \%(0 / 2)$ \\
\hline JAK-STAT pathway lesion & $21 \%(23 / 109)$ & $15 \%(7 / 47)$ & $0 \%(0 / 30)$ & $75 \%(12 / 16)^{* * * *}$ & $17 \%(1 / 6)$ & $20 \%(1 / 5)$ & $66 \%(2 / 3)$ & $0 \%(0 / 2)$ \\
\hline IKZFI deletion & $26 \%(27 / 103)$ & $12 \%(5 / 43)$ & $21 \%(6 / 28)$ & $69 \%(11 / 16)^{* * * *}$ & $17 \%(1 / 6)$ & $60 \%(3 / 5)$ & $33 \%(1 / 3)$ & $0 \%(0 / 2)$ \\
\hline IKZFI any aberration & $28 \%(29 / 103)$ & $16 \%(7 / 43)$ & $21 \%(6 / 28)$ & $69 \%(11 / 16)^{* * *}$ & $17 \%(1 / 6)$ & $60 \%(3 / 5)$ & $33 \%(1 / 3)$ & $0 \%(0 / 2)$ \\
\hline$\left.I K Z F\right|^{\text {plus }}$ & $14 \%(14 / 103)$ & $4 \%(4 / 43)$ & $0 \%(0 / 28)$ & $50 \%(8 / 16)^{* * *}$ & $0 \%(0 / 6)$ & $40 \%(2 / 5)$ & $0 \%(0 / 3)$ & $0 \%(0 / 2)$ \\
\hline trisomy 21 acquired & $8 \%(8 / 103)$ & $7 \%(3 / 43)$ & $4 \%(1 / 28)$ & $25 \%(4 / 16)^{*}$ & $0 \%(0 / 6)$ & $0 \%(0 / 5)$ & $0 \%(0 / 3)$ & $0 \%(0 / 2)$ \\
\hline trisomy 21 germline & $4 \%(4 / 110)$ & $2 \%(1 / 47)$ & $0 \%(0 / 30)$ & $19 \%(3 / 16)^{* *}$ & $0 \%(0 / 6)$ & $0 \%(0 / 5)$ & $0 \%(0 / 4)$ & $0 \%(0 / 2)$ \\
\hline EBF1 deletion & $6 \%(6 / 103)$ & $2 \%(1 / 43)$ & $0 \%(0 / 28)$ & $25 \%(4 / 16)^{* *}$ & $17 \%(1 / 6)$ & $0 \%(0 / 5)$ & $0 \%(0 / 3)$ & $0 \%(0 / 2)$ \\
\hline$K M T 2 D$ any aberration & $8 \%(9 / 109)$ & $2 \%(1 / 47)$ & $17 \%(5 / 30)$ & $0 \%(0 / 16)$ & $50 \%(3 / 6)^{* *}$ & $0 \%(0 / 5)$ & $0 \%(0 / 3)$ & $0 \%(0 / 2)$ \\
\hline SETD2 any aberration & $6 \%(6 / 109)$ & $6 \%(3 / 47)$ & $0 \%(0 / 30)$ & $6 \%(1 / 16)$ & $33 \%(2 / 6)^{*}$ & $0 \%(0 / 5)$ & $0 \%(0 / 3)$ & $0 \%(0 / 2)$ \\
\hline ETV6 any aberration & $17 \%(17 / 103)$ & $16 \%(7 / 43)$ & $4 \%(1 / 28)$ & $19 \%(3 / 16)$ & $0 \%(0 / 6)$ & $100 \%(5 / 5)^{* * * *}$ & $0 \%(0 / 3)$ & $50 \%(1 / 2)$ \\
\hline ETV6 deletion & $13 \%(13 / 103)$ & $14 \%(6 / 43)$ & $0 \%(0 / 28)$ & $6 \%(1 / 16)$ & $0 \%(0 / 6)$ & $100 \%(5 / 5)^{* * * *}$ & $0 \%(0 / 3)$ & $50 \%(1 / 2)$ \\
\hline ARPP21 deletion & $5 \%(5 / 103)$ & $5 \%(2 / 43)$ & $0 \%(0 / 28)$ & $6 \%(1 / 16)$ & $0 \%(0 / 6)$ & $40 \%(2 / 5)^{*}$ & $0 \%(0 / 3)$ & $0 \%(0 / 2)$ \\
\hline$B T G 1$ any aberration & $8 \%(8 / 103)$ & $7 \%(3 / 43)$ & $4 \%(1 / 28)$ & $6 \%(1 / 16)$ & $0 \%(0 / 6)$ & $60 \%(3 / 5)^{* *}$ & $0 \%(0 / 3)$ & $0 \%(0 / 2)$ \\
\hline HISTIH genes deletion & $7 \%(7 / 103)$ & $2 \%(1 / 43)$ & $0 \%(0 / 28)$ & $19 \%(3 / 16)$ & $0 \%(0 / 6)$ & $40 \%(2 / 5)^{*}$ & $33 \%(1 / 3)$ & $0 \%(0 / 2)$ \\
\hline
\end{tabular}

Only genetic lesions with uneven distribution across acute lymphoblastic leukemia (ALL) subtypes are included.The numbers of analyzed patients vary for individual genetic lesions based on the detection method: 103 patients were analyzed by single-nucleotide polymorphism array (SNPa), 109 were analyzed by RNA-sequencing in diagnostic sample, one patient who was analyzed by RNA-sequencing in relapse sample is not included in this analysis. 'Does not include $Z C C H C 7$-PAX5 fusion. ${ }^{2}$ Does not include uncommon type of $E R G$ del identified by SNPa but negative by polymerase chain reaction (PCR) in a single $D U X 4$ r-positive case. ${ }^{3}$ Includes $C R L F 2 r$ and $P 2 R Y 8$-CRLF2-low. ${ }^{*} P \leq 0.05$; * ${ }^{*} P \leq 0.01$; ${ }^{* *} P \leq 0.001 ;{ }^{* * *} P \leq 0.0001$; by Fisher Exact Probability test for the significant enrichment of genetic lesion in respective subtype compared to all remaining cases.

of BCR-ABL1-like and ETV6-RUNX1-like subtypes with respective genetic groups, we amended the cohort with two BCR-ABL1-positive and nine ETV6-RUNX1-positive ALLs. Eighteen patients had the BCR-ABL1-like phenotype according to HCA; two of them had IAMP21 and were not included in BCR-ABL1-like ALL for the subsequent analyses, and the remaining 16 patients were classified as $B C R$ - $A B L 1$-like ALL. Five patients were classified as ETV6-RUNX1-like ALL. Unlike DUX4r and ETV6RUNX1-positive/-like ALL, BCR-ABL1-like ALL did not co-cluster together in unsupervised HCA, confirming that the gene expression profile of $B C R$-ABL1(-like) ALL is less unique or specific (Figure 1D).

Overall, the most common B-other ALL subtype was DUX4r ALL (27\%), followed by BCR-ABL1-like (15\%), ZNF384r (5\%), ETV6-RUNX1-like (5\%), iAMP21 (4\%), and MEF2Dr ALL (2\%); the largest proportion of cases
("B-rest"; $n=47,43 \%$ ) did not belong to any of these subtypes (Figure 2).

Compared to remaining BCP-ALLs, B-others were more frequently males, older, had higher white blood cell (WBC) count, and worse early response to therapy (Online Supplementary Table S1). Analysis of demographic and clinical parameters in the two largest B-other-derived subtypes, DUX4r, BCR-ABL1-like, and B-rest subgroup showed a significantly higher proportion of females in $B C R$-ABL1-like compared to non-BCR-ABL1-like ALL, while there were no significant differences in age or WBC (Online Supplementary Table S1). Importantly, we found a significantly worse early response to treatment assessed by several criteria in the DUX4r ALL compared to non-DUX4r ALL, resulting in a higher proportion of highrisk patients among the DUX4r ALL (Table 1).

In total, 7 out of 110 B-other ALLs had an MPAL 


\begin{tabular}{|c|c|}
\hline Sex & \\
\hline Age & \\
\hline & imunophenotype \\
\hline & WBC \\
\hline & ednisone response \\
\hline & MRD d15 \\
\hline & R MRD risk \\
\hline & hal risk group \\
\hline & LL subtype \\
\hline & VAseq \\
\hline & IP array \\
\hline WE & ES \\
\hline & JX4 ncounts \\
\hline & $J \times 4 \mathrm{r}$ \\
\hline & $c(9 ; 20)$ \\
\hline & somy 21 \\
\hline & in $1 q$ \\
\hline & somy 5 \\
\hline & somy 18 \\
\hline & us $x$ \\
\hline & ZF1plus \\
\hline & $1 \times 5$ \\
\hline IKZ & $Z F 1$ \\
\hline$E R C$ & $G$ \\
\hline ETV & $v_{6}$ \\
\hline & ZLIXR1 \\
\hline & GG1 \\
\hline$E B F$ & $3 F 1$ \\
\hline TOX & $x$ \\
\hline LEF & $F 1$ \\
\hline & \\
\hline TCF & $F 3$ \\
\hline ZEB & \\
\hline & \\
\hline & RLF2 by FC \\
\hline & RLF2 \\
\hline$A B L$ & $3 L 1$ \\
\hline NR & RAS \\
\hline KRA & RAS \\
\hline FLT & T3 \\
\hline$J A K$ & $K 2$ \\
\hline JAK & K1 \\
\hline NF1 & \\
\hline & $12 B 3$ \\
\hline & PN11 \\
\hline 117 & $7 R$ \\
\hline$B R$ & \\
\hline & OKN2A \\
\hline & KKN2B \\
\hline & \\
\hline$C D N$ & KK6 \\
\hline$R B$ & \\
\hline & AT2D \\
\hline & TD2 \\
\hline & DEA \\
\hline & RID2 \\
\hline & TEBBP \\
\hline & $\begin{array}{l}200 / B T L A \\
\text { WD }\end{array}$ \\
\hline & ST1H genes \\
\hline & $33 C 2$ \\
\hline & $\begin{array}{l}33 C 1 \\
X 41 P\end{array}$ \\
\hline & $\begin{array}{l}x 41 P \\
D 3\end{array}$ \\
\hline & $\begin{array}{l}D P 3 \\
P P P 21\end{array}$ \\
\hline & PP10A \\
\hline & XOO1 \\
\hline & $\begin{array}{l}\text { REED1 } \\
\text { IIT }\end{array}$ \\
\hline & HTI \\
\hline
\end{tabular}
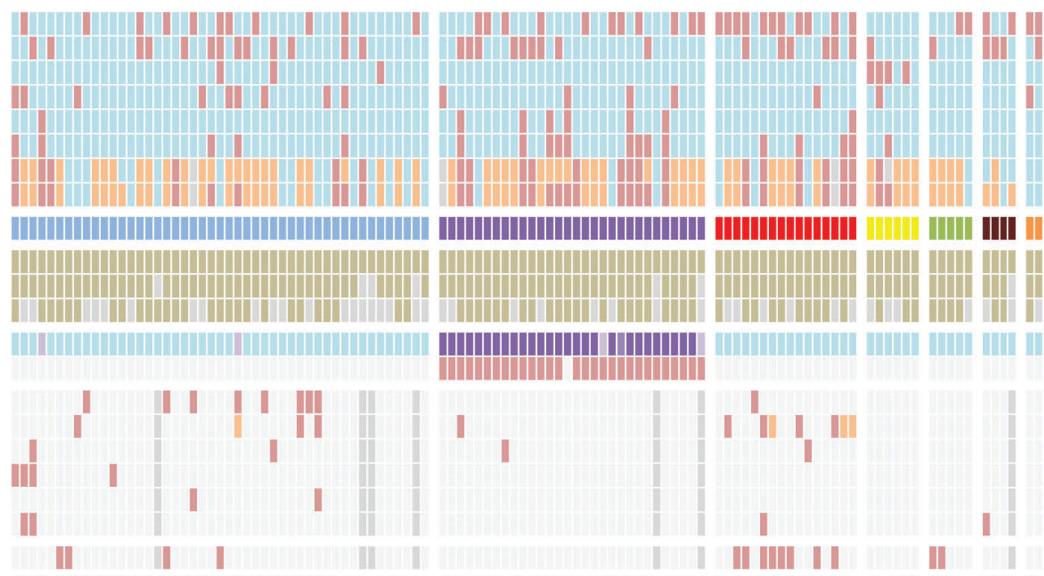

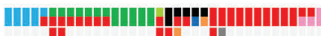

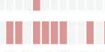
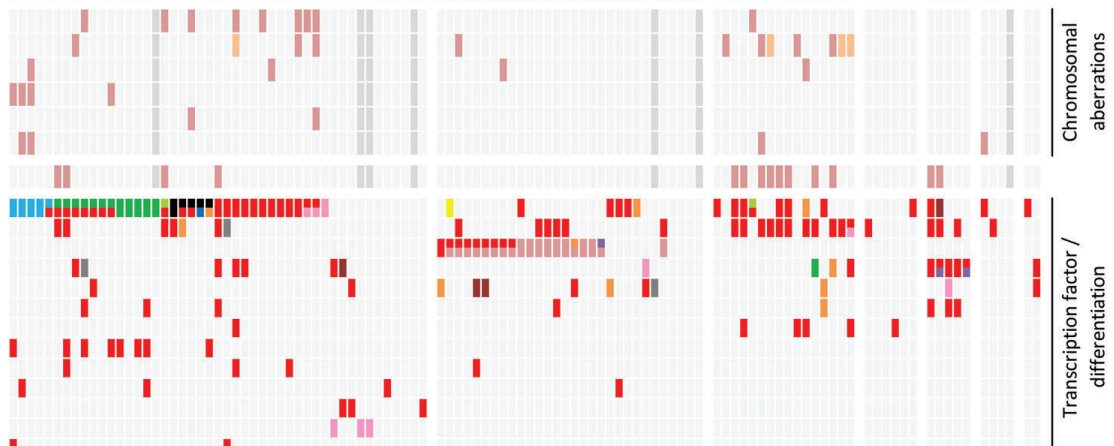

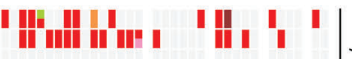
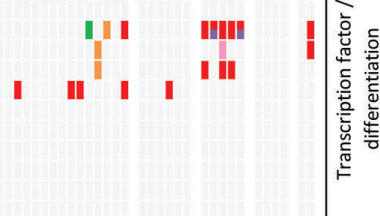

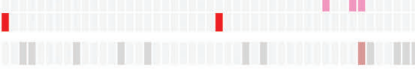

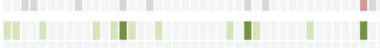

|| || | | || | || || || || || ||
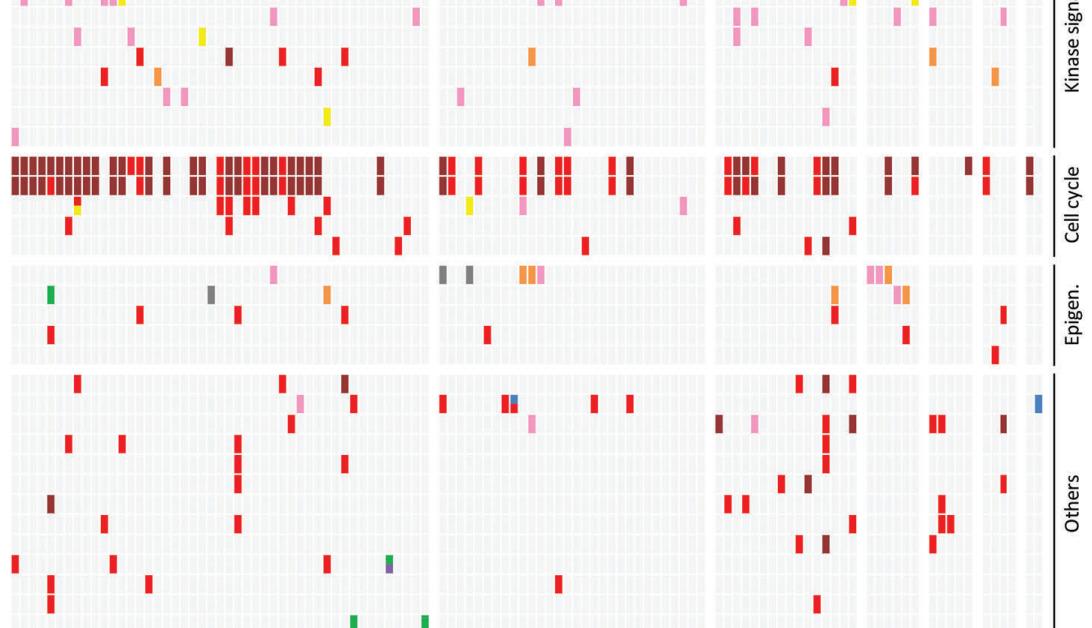

| | |||
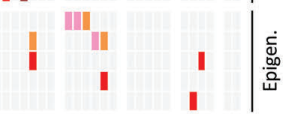

||||||||||| || ||| || || ||||
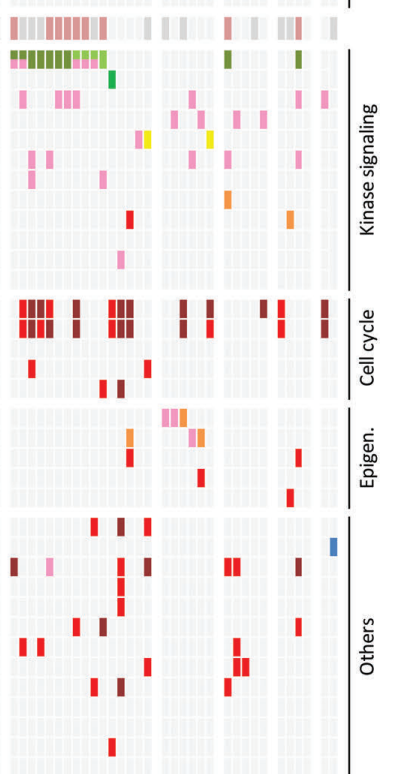

\begin{tabular}{|c|c|c|c|c|c|c|c|}
\hline Sex: & $\begin{array}{l}\text { Male } \\
\text { Female }\end{array}$ & All subtype: & $\begin{array}{l}\text { B-rest } \\
D U \times 4 \mathrm{r}\end{array}$ & 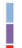 & IKZF1plus & $\begin{array}{l}\mathrm{Neg} \\
\text { Pos }\end{array}$ & ] \\
\hline \multirow[t]{2}{*}{ Age: } & $<10 y$ & & $\begin{array}{l}\text { BCR-ABLI-like } \\
\text { ZNF384r }\end{array}$ & I & \multirow{3}{*}{ CRLF2 by FC: } & NA & 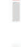 \\
\hline & $\geq 10 y$ & & ETVG-RUNX1-like & & & $\mathrm{Neg}$ & \\
\hline Immunophenotype: & $\begin{array}{l}\text { BCP } \\
\text { MPAL }\end{array}$ & & $\begin{array}{l}\text { IAMP21 } \\
\text { MEF2Dr }\end{array}$ & I & & $\begin{array}{l}\text { Pos } \\
\text { NA }\end{array}$ & ע \\
\hline Dg WBC: & $\begin{array}{l}<50.000 \\
\geq 50.000\end{array}$ & $\begin{array}{l}\text { RNAseq, } \\
\text { SNParray, WES: }\end{array}$ & $\begin{array}{l}\text { Performed } \\
\text { Not performed }\end{array}$ & I & \multirow[t]{4}{*}{$\begin{array}{l}\text { Genetic } \\
\text { aberration: }\end{array}$} & $\begin{array}{l}\text { Amplification } \\
\text { Biallelic deletion (SNPa) }\end{array}$ & 【 \\
\hline Prednisone respose: & $\begin{array}{l}\text { Good } \\
\text { Poor }\end{array}$ & DUX4 ncounts: & $\begin{array}{l}<100 \\
100-499\end{array}$ & & & $\begin{array}{l}\text { Monoallelic deletion (SNPa) } \\
\text { Deletion (PCR) } \\
\text { In-frame fusion }\end{array}$ & \\
\hline FC MRD d15: & $\begin{array}{l}<10 \% \\
\geq 10 \%\end{array}$ & & $\begin{array}{l}500-999 \\
\leq 1000\end{array}$ & I & & $\begin{array}{l}\text { Out-of-frame fusion } \\
\text { Missense mutation }\end{array}$ & \\
\hline \multirow[t]{2}{*}{$\begin{array}{l}\text { PCR MRD risk, final } \\
\text { risk group: }\end{array}$} & & DUX4r: & & I & & $\begin{array}{l}\text { Nonsense mutation } \\
\text { Frameshift mutation } \\
\text { In-frame indel }\end{array}$ & 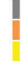 \\
\hline & $\begin{array}{l}\text { HR } \\
\text { NA }\end{array}$ & Trisomy 21: & $\begin{array}{l}\text { No } \\
\text { Yes - somatic } \\
\text { Yes - germline } \\
\text { NA }\end{array}$ & 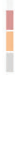 & $\begin{array}{l}\text { Specific genetic } \\
\text { aberration: }\end{array}$ & $\begin{array}{l}\text { PAXS5 AMP } \\
\text { PAX5 P8OR } \\
\text { ZCCHC7-PAX5 } \\
\text { IGH-CRLF2 } \\
\text { P2RY8-CRLF2 } \\
\text { P2RY8-CRLF2-IOW }\end{array}$ & 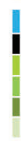 \\
\hline
\end{tabular}

Figure 3. Graphical overview of clinical characteristics, the analyses performed and the genetic findings in 110 B-other acute lymphoblastic leukemia (ALL) patients grouped by ALL subtype. Patients are ordered according to the ALL subtype; demographic and clinical data are shown above, while genetic data are displayed below the ALL subtype track. Recurrently affected genes are arranged according to functional categories. Epigen.: epigenetic regulators/modifiers; MPAL: BCP-myeloid mixed phenotype acute leukemia; Dg WBC; initial white blood cell count (cells/ $\mu$ L); y: years; FC: flow cytometry; SR: standard risk; MR: medium risk; HR: high risk; Neg: negative; Pos: positive; ncounts: normalized counts. For definitions of good and poor prednisone response, polymerase chain reaction (PCR) minimal residual disease (MRD) risk, and final risk group stratification see Table 1, Online Supplementary Methods and Online Supplementary Table S6. 


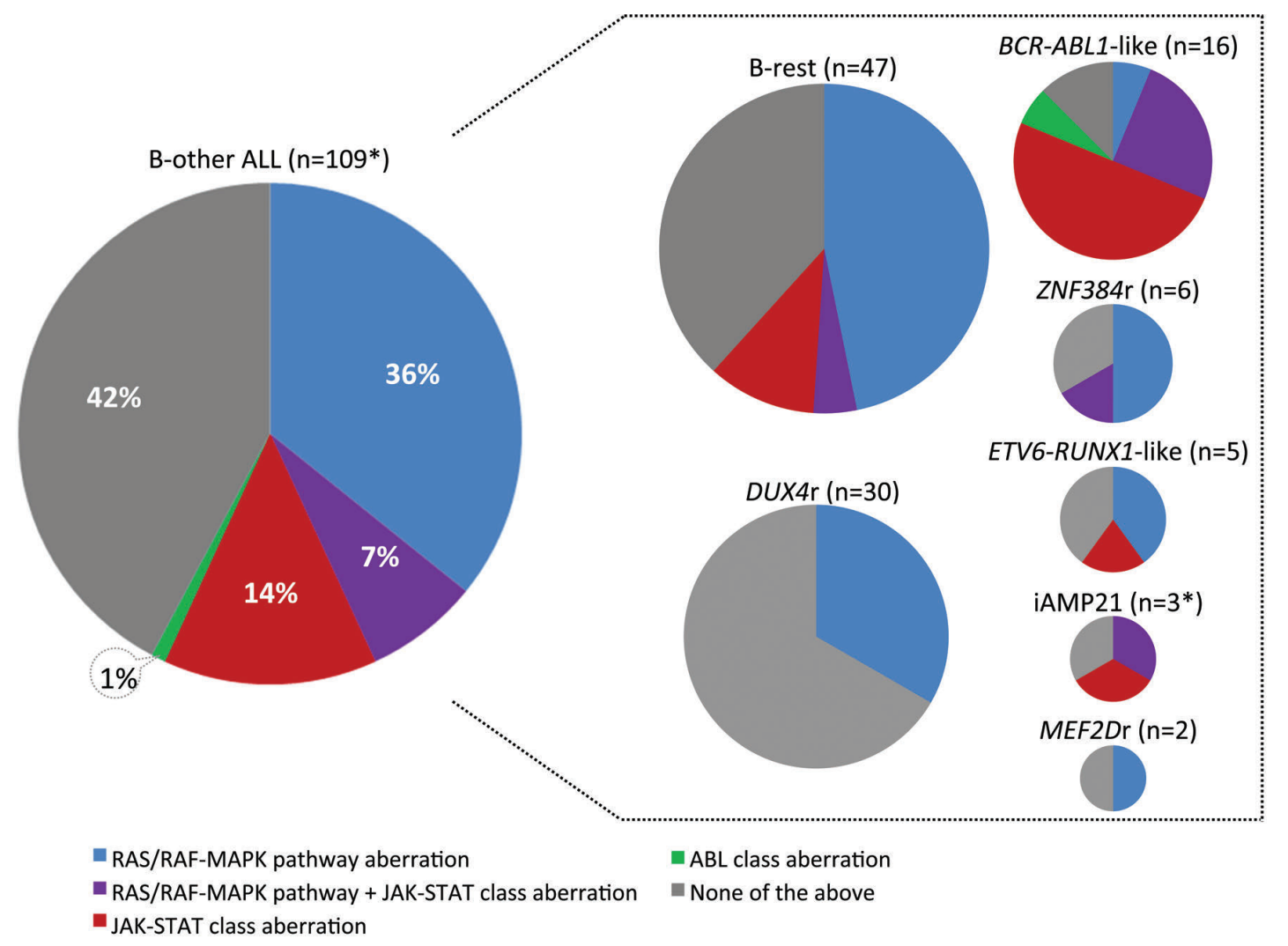

Figure 4. Frequency and distribution of kinase aberrations. The graphs are proportional to (sub)cohort sizes (number of patients in the total B-other cohort and in individual subtypes). *The one patient who was analyzed by RNA-sequencing only at relapse is not included in this analysis.

immunophenotype and these were significantly enriched in ZNF384r ALL [MPALs comprised 67\% (4 out of 6) of ZNF384r vs. $3 \%$ (3 out of 104) of non-ZNF384r ALLs; $P<0.0001]$.

\section{Genomic characterization of the study cohort}

Genomic aberrations were analyzed by SNPa, WES, RNA-sequencing and PCR. On average, we found 9 CNAs/UPDs per patient by SNPa (range 0-35) and 14 mutations [single nucleotide variants (SNVs), insertions/deletions (indels)] by WES (range 1-65) (Online Supplementary Tables S7 and S8). In total, we identified 18 recurrently (in $\geq 2$ patients) mutated genes by WES, and 43 recurrent chromosomal imbalances and CNAs subtracted to genes by SNPa; all had already been reported to occur in ALL. In addition to IGH-DUX4, we found 41 different fusion transcripts including 23 that were novel: 24 were inframe (13 out of 24 novel), 9 contained a full coding sequence of one partner gene ( 3 out of 9 novel), and 8 were out-of-frame (7 out of 8 novel) (Online Supplementary Table S9). Subclonal deletions of $E R G$ and P2RY8-CRLF2 fusions were detected by PCR in 11 and ten patients negative for these lesions by SNPa and RNA-sequencing, respectively. All recurrent findings are shown together with clinical parameters in Figure 3 and Online Supplementary Table S6.

\section{Specific genetic features of the B-other-derived acute lymphoblastic leukemia subtypes}

No significant differences were observed in numbers of CNAs/UPDs or SNVs/indels comparing individual ALL subtypes except for a significantly lower number of CNAs/UPDs in DUX4r versus remaining ALL cases $(4 \pm 0.5$ vs. $10 \pm 0.9 ; P<0.0001$ ) (Online Supplementary Figure S2). Apart from the MEF2Dr and iAMP21 ALL, which were represented by a very low number of patients, each of the remaining subtypes was significantly enriched for specific genetic aberrations (Table 2). ERGdel occurred in 63\% of DUX4r ALL but not in non-DUX4r ALL patients. Aberrations of TBLXR1 and DMD genes were also enriched in DUX4r ALL. BCR-ABL1-like ALL was enriched for the CRLF2 fusions, IKZF1del, both constitutional and somatic trisomy 21, and deletions of EBF1. Mutations in the KMT2D and SETD2 genes (encoding histone methyltransferases) were mutually exclusive and occurred in 5 out of 6 ZNF384r ALL patients. In agreement with our previous study, all ETV6RUNX1-like patients harbored an ETV6 aberration (5 out of 5 had a deletion and 2 out of 5 had a fusion). This subtype was also enriched for ARPP 21 deletions, deletions of histone gene cluster on 6p22.2, and BTG1 aberrations.

\section{Specific genetic features of the B-rest acute lymphoblastic leukemia subset}

The B-rest subset was significantly enriched for the PAX 5 fusions, PAX5 amplifications, PAX 5 P80R mutation, CDKN2A/B deletions, dic $(9 ; 20)$, and TOX deletions compared to remaining B-other ALLs. It is worthy of note that the PAX5 fusions, amplifications and P80R mutations and TOX deletions occurred exclusively in the B-rest subset; 6 out of 8 TOX deletions occurred together with the PAX5 fusions (Table 2). 
Table 3. PAX5-involving fusion genes.

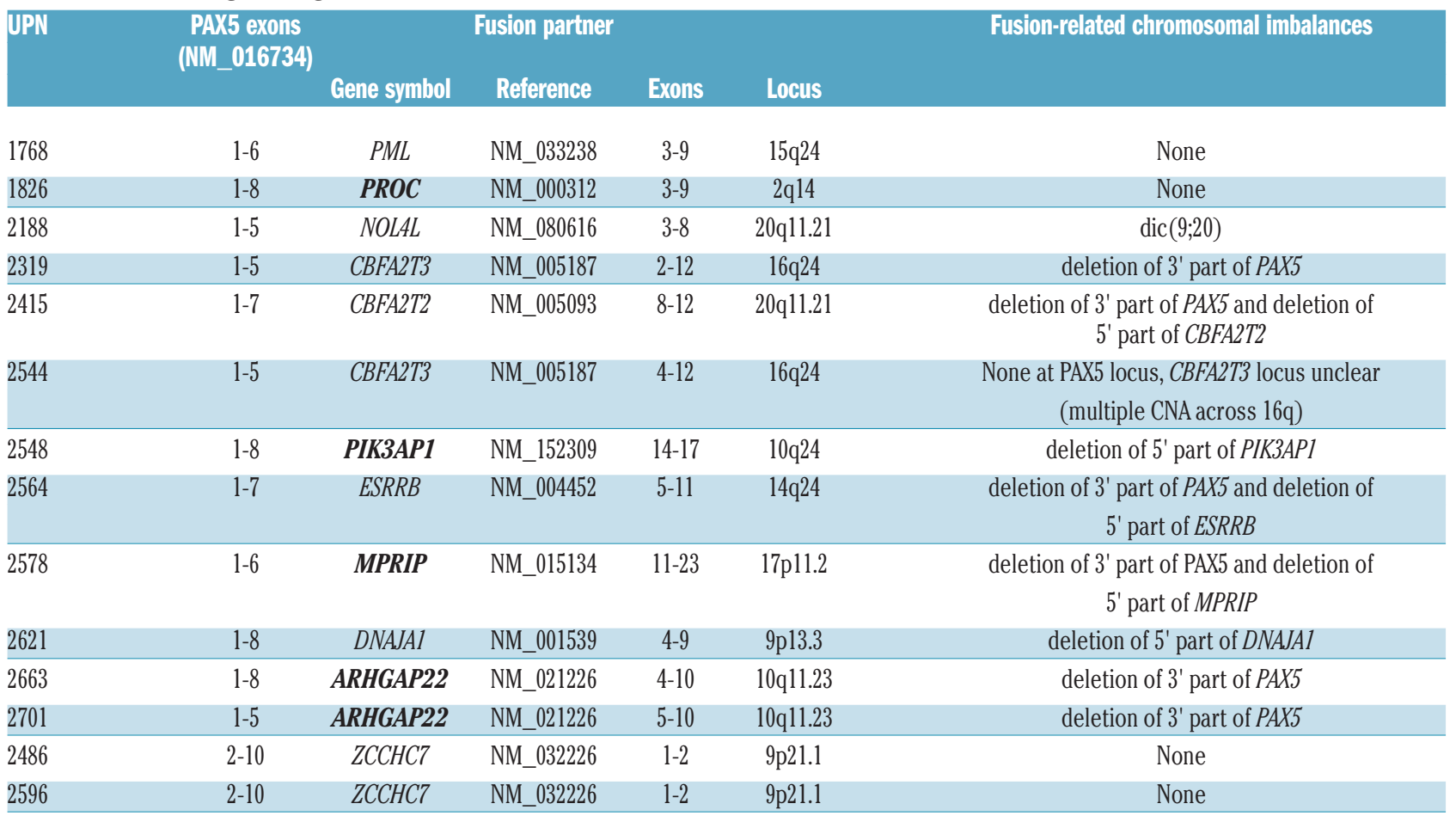

PAX5 fusion partners newly identified in the present study are shown in bold.

In total, we found 11 different $P A X 5$-involving in-frame fusions in 14 patients (Table 3). There were 2 types of fusions (Online Supplementary Figure S3). The more common type was comprised of the 5' part of PAX5 (exons 15/6/7/8) with the (partially) absent PAX5 transactivation domain (TAD), fused to various partner genes. This type was present in 12 patients, representing $11 \%$ of the total B-other cohort (Figure 2). The second type, represented only by the ZCCHC7-PAX5 fusion [generated by intrachromosomal inversion (Online Supplementary Figure S3)], involved the 3' part of PAX5 including both the DNAbinding domain and complete TAD (exons 2-10). We identified four novel PAX5 partner genes: ARHGAP22 (n=2), MPRIP, PIK3AP1, and PROC. To the best of our knowledge, except for PIK3AP1 (whose deletions were reported in adult $\mathrm{ALL}^{40}$ ), none of these genes have previously been reported as recurrently affected in BCP-ALL. Interestingly, we found rearrangement of the second PIK3AP1 allele resulting in an in-frame $P I K 3 A P 1-W D R 5$ fusion in the $P A X 5$-PIK3AP1-positive patient.

Intragenic $P A X 5$ amplification was found in six B-rest ALL patients, representing $5 \%$ of total B-other cohort (Figure 2). In five out of six patients, amplification encompassed exons $2-5(n=4)$ or exon $5(n=1)$, and thus corresponded to the recurrent type of amplification, PAX $5^{\mathrm{AMP}}$, which was recently characterized in detail; ${ }^{41}$ the remaining patient had atypical amplification encompassing exons 4-8 (Online Supplementary Figure S5). RNA-sequencing revealed aberrant $P A X 5$ transcripts containing amplified exons in all six patients (Online Supplementary Figure S6). In line with the previous study, ${ }^{41} P A X 5^{\mathrm{AMP}}$ was accompanied by the trisomy 5 in three out of five patients.

The PAX5 P80R mutation was found in five patients from the B-rest subset who co-clustered tightly together in unsupervised HCA (Figure 1D) and represented 5\% of the total B-other cohort (Figure 2). The variant allele frequency (VAF) of PAX5 P80R ranged from $32 \%$ to $100 \%$. The second PAX5 allele was deleted/lost in four out of five patients and likely inactivated in the remaining patient, with VAF $32 \%$ at the genomic but $98 \%$ at the mRNA level. We did not find any other genetic lesion which could explain co-clustering of these patients, which suggests that the specific expression signature was triggered by PAX5 P80R.

Unlike those with $P A X 5$ P80R, patients with $P A X 5$ fusions or $P A X 5^{\mathrm{AMP}}$ did not form clear clusters in unsupervised HCA; however, via an analysis of differential gene expression, we identified a gene set which distinguished patients with $P A X 5^{\mathrm{AMP}}$ from the remaining patients in supervised HCA (Online Supplementary Figure S7).

Two and three patients from the B-rest subset co-clustered together with ZNF384r and MEF2Dr ALL subtypes, respectively (Figure 1 and Online Supplementary Figure S7). We did not find any common genetic lesion in patients coclustering with ZNF384r ALL; however, two out of three patients co-clustering with MEF2Dr ALL harbored the ZNF618-NUTM1 fusion. The NUTM1-gene involving fusions were recently shown to occur recurrently in childhood ALL, outside the established ALL subtypes.

\section{Frequency and distribution of therapeutically relevant aberrations}

The $I K Z F 1^{\text {plus }}$ pattern was found in 14 out of 103 B-other ALLs (BCR-ABL1-like, $\mathrm{n}=8$; ETV6-RUNX1-like, $\mathrm{n}=2 ; \mathrm{B}$ rest ALLs, $\mathrm{n}=4)$ and was significantly enriched in the $B C R$ ABL1-like subtype (Table 2). It has been shown that $I K Z F 1^{\text {plus }}$ has a negative prognostic impact only in patients with detectable MRD at the end of induction. ${ }^{17}$ All but two 
$I K Z F 1^{\text {plus }}$-positive patients in the present study were MRD-positive at day 33 and thus predicted to have a higher risk of relapse.

The frequency and distribution of kinase aberrations is shown in Figure 4. Kinase aberrations that can be grouped into JAK/STAT-class were found in 23 patients who harbored lesions of CRLF2 ( $\mathrm{n}=21), J A K 2(\mathrm{n}=7), J A K 1 \quad(\mathrm{n}=6)$, $\operatorname{ILTR}(\mathrm{n}=2)$, and SH2B3 $(\mathrm{n}=1)$. The most common aberrations were CRLF2 gene rearrangements which had previously been shown to frequently occur at subclonal level. ${ }^{42}$ Accordingly, in addition to 12 P2RY8-CRLF2 and four IGH$C R L F 2$ fusions affecting a major leukemic clone (CRLF2r, $\mathrm{n}=16), P 2 R Y 8-C R L F 2$ fusions affecting a minor leukemic clone (P2RY8-CRLF2-low) were detected by RT-PCR in ten patients. The CRLF2r always resulted in surface CRLF2 positivity assessed by flow cytometry, was identified by RNA-sequencing, in the case of P2RY8-CRLF2 evidenced by PAR1 deletion on SNPa and was frequently accompanied by the activating CRLF2 mutation F232C (5 out of 16 cases) or by JAK $1 / 2$ mutation (6 out of 16 cases). On the other hand, all patients with P2RY8-CRLF2-low were negative for fusion transcript and PAR1 region deletion by RNA-sequencing and SNPa, respectively, and all but one were negative for surface CRLF2 by flow cytometry (a small CRLF2-positive subclone was reported in one patient). qPCR showed a clear separation ( $>1$ log distance) of the two groups of P2RY8-CLRF2-positive patients (Online Supplementary Figure S8). While CRLF2r (and JAK/STAT-class aberrations in general) were significantly associated with a BCR-ABL1-like subtype, P2RY8-CRLF2low occurred exclusively in the B-rest ALL subset (Table 2).

In the total B-other cohort, we found only one ABL-class aberration. The ETV6-ABL1 fusion was found in a BCR$A B L 1$-like ALL patient and was accompanied by another two in-frame fusions resulting from a single chromosomal rearrangement that we recently characterized in a separate report. $^{43}$

Aberrations activating RAS/RAF-MAPK signaling (aberrations of NRAS, KRAS, FLT3, BRAF, PTPN11, NF1) which may not be considered as suitable therapeutic targets because of their frequent subclonality, were found in 47 patients spread across all ALL subtypes.

Importantly, 50 out of 51 kinase aberrations detected by WES were also detected by RNA-sequencing.

\section{Discussion}

Although several large genomic studies have been published over the last few years, these were mostly based on non-consecutive cohorts of patients. ${ }^{4,6,7,21}$ Here, we present the first European population-based genomic study of pediatric B-other ALL based on a consecutive cohort of uniformly treated patients with a $100 \%$ inclusion rate. The only other consecutive study, the Swedish study by Lilljebjörn et al., ${ }^{2}$ based on 54 B-other ALLs (considering the $\mathrm{B}$-other definition used herein) with an inclusion rate of $69 \%$, showed a similarly high proportion of the "B-rest" ALL (40-45\%) and similarly infrequent ZNF384r and MEF2Dr ALL subgroups. Although the frequency of the remaining three subtypes differs between both studies (ETV6-RUNX1-like ALL 5\% vs. 11\%, DUX4r ALL 27\% vs. $15 \%, B C R-A B L 1$-like ALL $15 \%$ vs. $28 \%$ ), these differences are not statistically significant. Moreover, in the case of $B C R$ - $A B L 1$-like ALL, the difference might be influenced by heterogeneity in this subtype definition, which represents a broader phenomenon ${ }^{12}$ and reflects the low specificity of a $B C R-A B L 1$-positive/-like gene expression signature.

Despite the limitations of our analysis of clinical parameters imposed by the small size of the individual subtypes, we do present two important observations. First, we show a strong association of ZNF384r with mixed, B-myeloid immunophenotype. Although this association had already been established, ${ }^{5,44-46}$ here we demonstrate that ZNF384r ALL represents the majority of all consecutively diagnosed non-infant pediatric B-other B-myeloid MPALs (4 out of 7; 57\%). Second, we show a significant association of DUX4r ALL with worse early response to therapy. This ALL subtype is now often considered equivalent to ERGdel-positive ALL. However, an important proportion of DUX4r ALL lacks the ERGdel. We have shown previously that despite the association of ERGdel with worse early therapy response, this did not translate into worse outcome in the MRD-based BFM ALL 2000 protocol. $^{31}$ Although two recent studies reported a favorable outcome of DUX4r ALL, ${ }^{2,4}$ its potential heterogeneity with respect to ERGdel has still not been studied. Thus, the outcome of DUX4r ALL in BFM studies, both in general and stratified by ERGdel, still remains to be addressed.

Previous studies demonstrated the frequent occurrence and remarkable diversity of $P A X 5$ aberrations in childhood BCP ALL. ${ }^{9,47-49}$ We focused here on three types of $P A X 5$ aberrations that, in our study, were mutually exclusive with established ALL subtypes and altogether represented a large proportion of B-other ALL (20\%). Moreover, we suggest here that one of them, the PAX5 P80R mutation, likely represents a primary lesion that defines a novel biological ALL subtype, whose existence and character, however, remain to be confirmed and defined in detail by future studies. Among PAX5 fusions, we identified four novel PAX5 fusion partners further broadening an already sizeable $P A X 5$ recombinome..$^{50}$ Although the PAX5 fusions in our study lacked a specific gene expression signature, which questions their potential to define a unique biological subset, with respect to their frequency (which exceeds that of some already established subtypes) future efforts to evaluate their clinical impact are to be expected. Interestingly, the patients with $P A X 5$ fusions included in our study had a significantly better early treatment response compared to the other patients; none was stratified as high risk (Online Supplementary Table S10).

$I K Z F 1^{\text {plus }}$ genotype has been recently described to confer poor outcome (30-40\% 5-year event-free survival) in nonstandard risk patients on the ALL BFM 2000 study. ${ }^{17} \mathrm{We}$ have identified $14 I K Z F 1^{\text {plus }}$-positive patients, representing $14 \%$ of B-other ALLs. Because BCR-ABL1-like ALL is enriched for $I K Z F 1$ del and PAR1 deletion (included in the $I K Z F 1^{\text {plus }}$ definition), it has been assumed that $I K Z F 1^{\text {plus }}$ and $B C R-A B L 1$-like cases may largely overlap, although this has still not been demonstrated. In our study, $57 \%$ of patients with the $I K Z F 1^{\text {plus }}$ genotype were classified as $B C R-A B L 1$-like. Thus, although $I K Z F 1^{\text {plus }}$ was significantly enriched in $B C R-A B L 1$-like ALL, a large proportion of $I K Z F 1^{\text {plus }}$-positive patients were still not classified into this subtype. Only 2 out of $14 I K Z F 1^{\text {plus }}$-positive ALLs were stratified into standard risk, both belonging to the B-rest subset. Larger cohorts will be needed to determine whether a refined ALL subtype classification could help to 
further specify the prognostic impact of $I K Z F 1^{\text {plus }}$ and explain its absence in standard risk patients. ${ }^{17}$

One of the most striking findings of our study is the low frequency of kinase/cytokine receptor gene fusions other than those involving CRLF2. Except for CRLF2 fusions and a single case with ABL-class fusion, no other kinase-activating fusions were detected among all B-other patients diagnosed in the Czech Republic over a 7-year period. The low frequency of non-CRLF2 fusions, supported also by the result of the Swedish study, ${ }^{2}$ seems to contrast with some American genomic studies; ${ }^{21,30}$ however, a direct comparison of frequencies between the European and American populations is not possible at the moment, as only the two European studies refer to consecutive, unselected cohorts. Considering the low costs and the excellent performance of flow cytometry in detecting CRLF2r-positive patients, it remains questionable whether any benefit can be gained from testing the $B C R-A B L 1$-like signature in all B-other ALL patients (irrespective of their treatment response) in order to identify patients with a higher probability of having druggable kinase/cytokine receptor gene fusions. Instead, identification of targetable lesions (guiding the choice of appropriate drug) can be performed in patients with poor response to treatment upfront and irrespective of potential presence/absence of $B C R-A B L 1$-like phenotype.

In our study, we also aimed to assess the feasibility and benefit of incorporating genome-wide technologies into routine diagnostics. Thanks to centralization in a single laboratory, we successfully standardized all processes and minimized the time to integrate results of all analyses. Although we see some benefit in performing all three genome-wide analyses (SNPa, RNA-sequencing, WES) to obtain the most complex genomic profile, considering the cost-benefit ratio, we found the combination of SNPa and RNA-sequencing to be the most efficient. This combination enables the therapeutically most relevant aberrations (IKZF1 $1^{\text {plus }}$ and kinase-activating lesions) to be detected.
Moreover, RNA-sequencing enables patients to be classified into novel subtypes; this would not be possible with targeted approaches, which are methodologically and analytically less demanding and possibly cheaper. However, these advantages fade with the increasing number of aberrations that need to be tested and, in addition, undescribed/private, but druggable aberrations, cannot always be detected. Furthermore, the complex data gained through genome-wide methods are extremely valuable for retrospective discovery/validation analyses. Nevertheless, it should be noted that genome-wide methods also have additional limitations (e.g. lower sensitivity in fusion transcript detection than PCR), and these need to be considered and tested during the implementation process.

In conclusion, our study shows unbiased European population-based frequency of novel B-other ALL subtypes, recurrent (cyto)genetic aberrations as well as their mutual associations, some of which have not yet been reported. We believe that these findings not only help to strengthen and deepen the current knowledge of B-other ALL, but also provide an objective basis on which other groups/countries can decide how to optimize their current diagnostic routine.

\section{Acknowledgments}

We would like to thank to all centers of the Czech Paediatric Haematology Working Group (CPH).

\section{Funding}

This study was supported by the "Kapka nadeje" foundation, grants from the Czech Health Research Council (NV1530626A), Czech Science Foundation (GJ15-06049Y) and Charles University (Primus/MED/28, UNCE 204012) and by the project (Ministry of Health, Czech Republic) for conceptual development of research organization 00064203 (University Hospital Motol, Prague, Czech Republic). Research infrastructure was supported by the Ministry of Education, Youth and Sports (NPU I no. LO1604 and LM2015091).

\section{References}

1. Rand V, Parker H, Russell LJ, et al. Genomic characterization implicates iAMP21 as a likely primary genetic event in childhood Bcell precursor acute lymphoblastic leukemia. Blood. 2011;117(25):6848-6855.

2. Lilljebjorn $\mathrm{H}$, Henningsson $\mathrm{R}$, HyreniusWittsten $\mathrm{A}$, et al. Identification of ETV6RUNX1-like and DUX4-rearranged subtypes in paediatric B-cell precursor acute lymphoblastic leukaemia. Nat Commun. 2016;7:11790.

3. Yasuda T, Tsuzuki S, Kawazu M, et al. Recurrent DUX4 fusions in B cell acute lymphoblastic leukemia of adolescents and young adults. Nat Genet. 2016;48(5):569-574.

4. Zhang J, McCastlain $\mathrm{K}$, Yoshihara $\mathrm{H}$, et al. Deregulation of DUX4 and ERG in acute lymphoblastic leukemia. Nat Genet. 2016; 48(12):1481-1489.

5. Hirabayashi S, Ohki K, Nakabayashi K, et al. ZNF384-related fusion genes define a subgroup of childhood B-cell precursor acute lymphoblastic leukemia with a characteristic immunotype. Haematologica. 2017;102 (1):118-129.
6. Liu YF, Wang BY, Zhang WN, et al. Genomic Profiling of Adult and Pediatric B-cell Acute Lymphoblastic Leukemia. EBioMedicine. 2016;8:173-183.

7. Gu Z, Churchman M, Roberts $K$, et al. Genomic analyses identify recurrent MEF2D fusions in acute lymphoblastic leukaemia. Nat Commun. 2016;7:13331.

8. Den Boer ML, van Slegtenhorst M, De Menezes RX, et al. A subtype of childhood acute lymphoblastic leukaemia with poor treatment outcome: a genome-wide classification study. Lancet Oncol. 2009; 10(2):125 134.

9. Mullighan CG, Su X, Zhang J, et al. Deletion of IKZF1 and prognosis in acute lymphoblastic leukemia. $N$ Engl J Med. 2009;360(5):470-480.

10. Roberts KG, Morin RD, Zhang J, et al Genetic alterations activating kinase and cytokine receptor signaling in high-risk acute lymphoblastic leukemia. Cancer Cell. 2012;22(2):153-166

11. Zaliova M, Kotrova M, Bresolin S, et al. ETV6/RUNX1-like acute lymphoblastic leukemia: A novel B-cell precursor leukemia subtype associated with the CD27/CD44 immunophenotype. Genes Chromosomes Cancer. 2017;56(8):608-616.

12. Boer JM, Marchante JR, Evans WE, et al. BCR-ABL1-like cases in pediatric acute lymphoblastic leukemia: a comparison between DCOG/Erasmus MC and COG/St. Jude signatures. Haematologica. 2015;100(9):e354357

13. Clappier E, Grardel N, Bakkus M, et al. IKZF1 deletion is an independent prognostic marker in childhood B-cell precursor acute lymphoblastic leukemia, and distinguishes patients benefiting from pulses during maintenance therapy: results of the EORTC Children's Leukemia Group study 58951. Leukemia. 2015;29(11):2154-2161

14. Dorge P, Meissner B, Zimmermann M, et al IKZF1 deletion is an independent predictor of outcome in pediatric acute lymphoblastic leukemia treated according to the ALL-BFM 2000 protocol. Haematologica. 2013;98(3):428-32.

15. Kuiper RP, Waanders E, van der Velden VH, et al. IKZF1 deletions predict relapse in uniformly treated pediatric precursor B-ALL. Leukemia. 2010;24(7):1258-1264.

16. Boer JM, van der Veer A, Rizopoulos D, et al 
Prognostic value of rare IKZF1 deletion in childhood B-cell precursor acute lymphoblastic leukemia: an international collaborative study. Leukemia. 2016;30(1):32-38.

17. Stanulla M, Dagdan E, Zaliova M, et al. IKZF1(plus) Defines a New Minimal Residual Disease-Dependent Very-Poor Prognostic Profile in Pediatric B-Cell Precursor Acute Lymphoblastic Leukemia. J Clin Oncol. 2018:36(12):1240-1249.

18. Hertzberg L, Vendramini E, Ganmore I, et al. Down syndrome acute lymphoblastic leukemia, a highly heterogeneous disease in which aberrant expression of CRLF2 is associated with mutated JAK2: a report from the International BFM Study Group. Blood. 2010;115(5):1006-1017.

19. Mullighan CG, Collins-Underwood JR, Phillips LA, et al. Rearrangement of CRLF2 in B-progenitor- and Down syndrome-associated acute lymphoblastic leukemia. Nat Genet. 2009;41(11):1243-1246.

20. Perentesis JP, Bhatia S, Boyle E, et al. RAS oncogene mutations and outcome of therapy for childhood acute lymphoblastic leukemia. Leukemia. 2004;18(4):685-692.

21. Roberts KG, Li Y, Payne-Turner D, et al. Targetable kinase-activating lesions in Phlike acute lymphoblastic leukemia. N Engl J Med. 2014;371(11):1005-1015.

22. Russell LJ, Capasso M, Vater I, et al. Deregulated expression of cytokine receptor gene, CRLF2, is involved in lymphoid transformation in B-cell precursor acute lymphoblastic leukemia. Blood. 2009; 114(13):2688-2698.

23. Zhang J, Mullighan CG, Harvey RC, et al. Key pathways are frequently mutated in high-risk childhood acute lymphoblastic leukemia: a report from the Children's Oncology Group. Blood. 2011; 118(11):3080-3087.

24. Holmfeldt L, Wei L, Diaz-Flores E, et al. The genomic landscape of hypodiploid acute lymphoblastic leukemia. Nat Genet. 2013;45(3):242-252.

25. Paulsson K, Lilljebjorn H, Biloglav A, et al. The genomic landscape of high hyperdiploid childhood acute lymphoblastic leukemia. Nat Genet. 2015;47(6):672-676.

26. van $\operatorname{der}$ Veer A, Waanders E, Pieters R, et al. Independent prognostic value of BCR-ABL1like signature and IKZF1 deletion, but not high CRLF2 expression, in children with Bcell precursor ALL. Blood. 2013; 122(15):2622-2629.
27. Andersson AK, Ma J, Wang J, et al. The landscape of somatic mutations in infant MLLrearranged acute lymphoblastic leukemias. Nat Genet. 2015:47(4):330-337.

28. Boer JM, Steeghs EM, Marchante JR, et al. Tyrosine kinase fusion genes in pediatric BCR-ABL1-like acute lymphoblastic leukemia. Oncotarget. 2017;8(3):4618-4628.

29. Zaliova M, Moorman AV, Cazzaniga G, et al. Characterization of leukemias with ETV6-ABL1 fusion. Haematologica. 2016; 101(9):1082-1093

30. Reshmi SC, Harvey RC, Roberts KG, et al Targetable kinase gene fusions in high-risk B-ALL: a study from the Children's Oncology Group. Blood. 2017; 129(25):3352-3361.

31. Zaliova M, Zimmermannova O, Dorge P, et al. ERG deletion is associated with $C D 2$ and attenuates the negative impact of IKZF1 deletion in childhood acute lymphoblastic leukemia. Leukemia. 2014; 28(1):182-185.

32. Clappier E, Auclerc MF, Rapion J, et al. An intragenic ERG deletion is a marker of an oncogenic subtype of B-cell precursor acute lymphoblastic leukemia with a favorable outcome despite frequent IKZF1 deletions. Leukemia. 2014;28(1):70-77.

33. Kim D, Salzberg SL. TopHat-Fusion: an algorithm for discovery of novel fusion transcripts. Genome Biol. 2011;12(8):R72.

34. McPherson A, Hormozdiari F, Zayed A, et al. deFuse: an algorithm for gene fusion discovery in tumor RNA-Seq data. PLoS Comput Biol. 2011;7(5):e1001138.

35. Li H, Durbin R. Fast and accurate long-read alignment with Burrows-Wheeler transform. Bioinformatics. 2010;26(5):589-595

36. Dobin A, Davis CA, Schlesinger F, et al. STAR: ultrafast universal RNA-seq aligner. Bioinformatics. 2013;29(1):15-21.

37. Koboldt DC, Zhang $\mathrm{Q}$, Larson DE, et al. VarScan 2: somatic mutation and copy number alteration discovery in cancer by exome sequencing. Genome Res. 2012; 22(3):568576.

38. Anders S, McCarthy DJ, Chen Y, et al. Count-based differential expression analysis of RNA sequencing data using $R$ and Bioconductor. Nat Protoc. 2013;8(9):17651786

39. Love MI, Huber W, Anders S. Moderated estimation of fold change and dispersion for RNA-seq data with DESeq2. Genome Biol. 2014;15(12):550

40. Safavi S, Hansson M, Karlsson K, Biloglav A
Johansson B, Paulsson K. Novel gene targets detected by genomic profiling in a consecutive series of 126 adults with acute lymphoblastic leukemia. Haematologica. 2015;100(1):55-61.

41. Schwab C, Nebral K, Chilton L, et al. Intragenic amplification of PAX5: a novel subgroup in B-cell precursor acute lymphoblastic leukemia? Blood Adv. 2017; 1(19):1473-1477.

42. Morak M, Attarbaschi A, Fischer S, et al Small sizes and indolent evolutionary dynamics challenge the potential role of P2RY8-CRLF2-harboring clones as main relapse-driving force in childhood ALL. Blood. 2012;120(26):5134-5142.

43. Lukes J, Potuckova E, Sramkova L, et al. Two novel fusion genes, AIF1L-ETV6 and ABL1 AIF1L, result together with ETV6-ABL1 from a single chromosomal rearrangement in acute lymphoblastic leukemia with prenatal origin. Genes Chromosomes Cancer. 2018.57(9):471-477.

44. Gocho Y, Kiyokawa N, Ichikawa H, et al. A novel recurrent EP300-ZNF384 gene fusion in B-cell precursor acute lymphoblastic leukemia. Leukemia. 2015;29(12):2445 2448.

45. Shago M, Abla O, Hitzler J, Weitzman S, Abdelhaleem M. Frequency and outcome of pediatric acute lymphoblastic leukemia with ZNF384 gene rearrangements including a novel translocation resulting in an ARID1B/ZNF384 gene fusion. Pediatr Blood Cancer. 2016:63(11):1915-1921.

46. Alexander TB, Gu Z, Iacobucci I, et al. The genetic basis and cell of origin of mixed phenotype acute leukaemia. Nature. 2018; 562(7727):373-379.

47. Coyaud E, Struski S, Prade N, et al. Wide diversity of PAX5 alterations in B-ALL: Groupe Francophone de Cytogenetique Hematologique study. Blood. 2010; 115(15):3089-3097.

48. Nebral K, Denk D, Attarbaschi A, et al. Incidence and diversity of PAX5 fusion genes in childhood acute lymphoblastic leukemia. Leukemia. 2009;23(1):134-143.

49. Stasevich I, Inglott S, Austin N, et al. PAX5 alterations in genetically unclassified childhood Precursor B-cell acute lymphoblastic leukaemia. Br J Haematol. 2015;171(2):263272.

50. Atlas of Genetics and Cytogenetics in Oncology and Haematology. Available from: http://AtlasGenetics Oncology.org 